\title{
The approach to fracture diagnosis by means of experimental measurements of the stored energy
}

\author{
A. Vedernikova, A. Iziumova, A. Vshivkov, O. Plekhov \\ Institute of Continuous Media Mechanics UB RAS, 614013 Perm, Russia \\ terekbina.a@icmm.ru
}

\begin{abstract}
Energy dissipation in metals under irreversible deformation leads to intensive heat generation in strain localization zones. In this work, we focus on measuring heat source power using temperature data obtained by IR thermography. The calculated heat source power data were verified by analyzing the data recorded by the Seebeck effect-based heat flux sensor developed in previous study. Quasi-static tensile tests were conducted on titanium alloy Grade 2 flat specimens. It is shown that the thermography data and the results obtained with the heat flux sensor are in good quantitative agreement. The dependence of the moment of fracture of metal specimens on the change in localized heat generation caused by irreversible deformation was determined.
\end{abstract}

KEYWORDS. Stored energy; Thermography; Heat flux sensor.

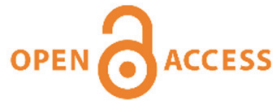

Citation: Vedernikova, A., Iziumova, A., Vshivkov, A., Plekhov, O., The approach to fracture diagnosis by means of experimental measurements of the stored energy, Frattura ed Integrità Strutturale, 49 (2019) 314-320.

Received: 04.04.2019

Accepted: 04.06.2019

Published: 01.07.2019

Copyright: (C) 2019 This is an open access article under the terms of the CC-BY 4.0, which permits unrestricted use, distribution, and reproduction in any medium, provided the original author and source are credited.

\section{INTRODUCTION}

7 oday the investigations of many authors [1-8] are aimed at creating theoretical models describing the behavior of materials under deformations process. These models include thermodynamic and structural parameters that determine the deformation process. The energy dissipation in metals during irreversible deformation and the change in the internal structure lead to intensive heat generation in strain localization zones. Modern IR detectors allow studying the evolution of energy accumulation and dissipation and provide a deeper insight into plastic-deformation localization mechanisms [1-5]. In practice, researchers often differ in the quantitative estimation of the dissipation heat and the stored energy in a cold-worked material, which impedes the development of methods of damage assessment [6].

In this study, the dissipative properties of Grade 2 titanium alloys were investigated in quasi-static tensile tests. The infrared thermography technique (IRT) and the contact heat flux sensor used to study the evolution of heat sources and determine the critical state of materials. Analysis of the results of investigation on the energy balance in the fracture zone obtained with the infrared techniques and heat flux sensor during the deformation of titanium alloy Grade 2 lend confirm the validity of the proposed method. 


\section{EXPERIMENTAL DETAILS}

7 he standard quasistatic tensile tests were performed on titanium alloy Grade 2 flat specimens. The chemical composition of the material is presented in the Tab. 1. Specimens (gauge length of $120 \mathrm{~mm}$ and width of $20 \mathrm{~mm}$ ) were made of a $3 \mathrm{~mm}$ thick sheet. Mechanical tests were carried out using a $300 \mathrm{kN}$ electromechanical testing machine Shimadzu AG-X Plus. The geometry of specimens and the deformation curve are shown in Fig. 1. Investigation of the heat source evolution was carried out using the temperature data obtained by an infrared camera FLIR SC 5000. IR camera has the following features: the spectral range of 3-5 $\mu \mathrm{m}$, the maximum frame size is $320 \times 256$ pixels, the spatial resolution is $10^{-4}$ meters. The temperature sensitivity is in the range from $25 \mathrm{mK}$ to $300 \mathrm{~K}$. The surface of the specimens intended for infrared shooting was polished in several stages and coated by a thin layer of amorphous carbon to enhance the emissivity.

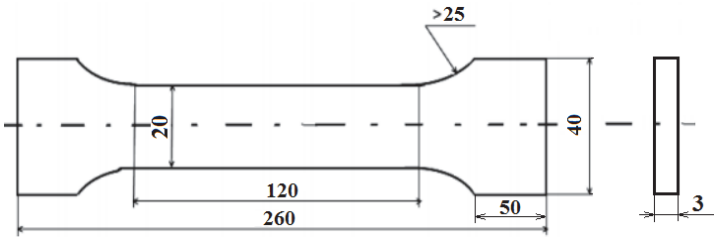

a)

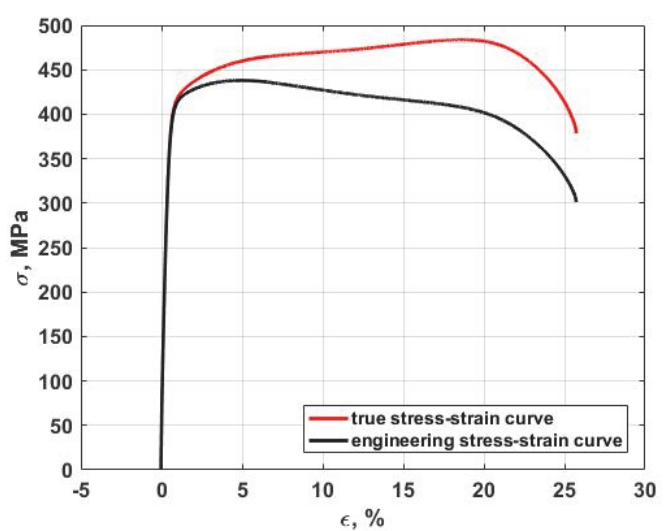

b)

Figure 1: (a) Geometry of specimens; (b) Deformation curve.

\begin{tabular}{cccccccc}
\hline $\mathrm{Fe}$ & $\mathrm{C}$ & $\mathrm{Si}$ & $\mathrm{N}$ & $\mathrm{Ti}$ & $\mathrm{O}$ & $\mathrm{H}$ & Other \\
0.25 & 0.07 & 0.1 & 0.04 & $99.24-99.7$ & 0.2 & 0.01 & 0.3 \\
\hline
\end{tabular}

Table 1: The chemical composition of titanium alloy Grade 2.

To verify the heat source power obtained by the infrared technique, the contact heat flux sensor based on the Seebeck effect [9] was directly attached to the specimen. To enhance the heat flow, a heat-conductive paste was applied between the sensor and the specimen.

\section{EVALUATION OF THE STORED ENERGY}

$\mathrm{W}$

e calculate the heat source field by applying the heat conduction Eqn. (1) to infrared thermography data:

$$
\rho c \frac{\partial T(x, y, z, t)}{\partial t}=Q(x, y, z, t)+k\left(\frac{\partial^{2} T(x, y, z, t)}{\partial x^{2}}+\frac{\partial^{2} T(x, y, z, t)}{\partial y^{2}}+\frac{\partial^{2} T(x, y, z, t)}{\partial z^{2}}\right)
$$

where $T(x, y, z, t)$ is the temperature field, $\rho$ is the material density $(4505 \mathrm{~kg} / \mathrm{m} 3), c$ is the heat capacity $(540 \mathrm{~J} /(\mathrm{kg} \cdot \mathrm{K}))$, $k$ is the heat conductivity $(16.2 \mathrm{~W} /(\mathrm{m} \cdot \mathrm{K})), Q(x, y, z, t)$ is the heat source field, $x, y, z$ are the coordinates, and $t$ is the time.

The infrared camera allows registering the temperature distribution over the specimen surface but not across the specimen thickness. That is the reason why rather thin specimens are used in experimental investigations. It makes possible to assume that the temperature distribution through the specimen thickness is homogeneous. 
The volume-averaged Eqn. (1) is used for estimation of the integral power of the heat source. To this end, a standard averaging procedure was conducted. The difference between the averaged specimen temperature on volume $T$ and the initial specimen temperature $T_{0}$ in the thermal balance with the environment is defined as:

$$
\theta^{\prime}(t)=\frac{1}{V} \int_{-a / 2}^{a / 2} \int_{-b / 2}^{b / 2} \int_{-b / 2}^{b / 2}\left(T(x, y, z, t)-T_{0}\right) d x d y d z=\theta(t)-T_{0},
$$

where $a, b, b$ are the length, width and thickness of the specimen, respectively, and $V$ is the volume.

The boundary conditions are expressed as follows:

$$
\begin{aligned}
& \left.\frac{\partial T(x, y, \mathrm{z}, t)}{\partial x}\right|_{x=\frac{a}{2}}=-\left.\frac{\partial T(x, y, \mathrm{z}, t)}{\partial x}\right|_{x=-\frac{a}{2}}, \\
& -\left.k \frac{\partial T(x, y, \mathrm{z}, t)}{\partial x}\right|_{x=\frac{a}{2}}=\frac{g_{x}}{a} \int_{-a / 2}^{a / 2}\left(T(x, y, z, t)-T_{0}\right) d x d y d z,
\end{aligned}
$$

where $g_{x}$ means the heat exchange coefficient between the specimen and the environment on the corresponding edge of the specimen.

Therefore, integrating Eqn. (1), considering expressions (2) and boundary conditions (3), we obtain relation (4) to estimate the heat source field caused by irreversible deformation:

$$
S(t)=m c \frac{\partial \theta(t)}{\partial t}+\beta V\left(\theta(t)-T_{0}\right)
$$

where $\theta$ is the average temperature of the examined surface, $m$ is the mass of the representative volume where the average temperature is taken from, $S(t)$ is the heat source field $(\mathrm{W})$, and $\beta$ is the material parameter that determines heat losses associated with the heat exchange with the environment.

The parameter $\beta$ is determined by the additional experiment data from the tests in which the specimen was cooled after pulse point heating. Fig. 2 a presents the IR image, which was made during the pulse point heating experiment, and data of the average temperature of the heating area during the cooling process. To calculate the constant $\beta$, it is necessary to approximate the experimental data of the average specimen temperature after pulse point heating in terms of the solution of the averaged thermal conductivity Eqn. (4) with a zero source (Fig. 2b). The experimentally obtained value of parameter $\beta$ is equal to $3.65 \cdot 10^{4}$.

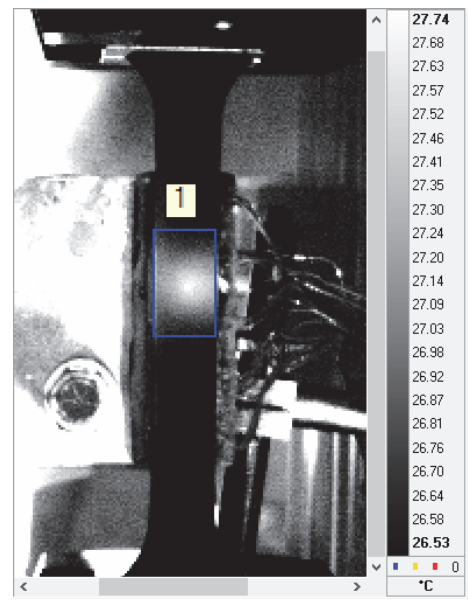

a)

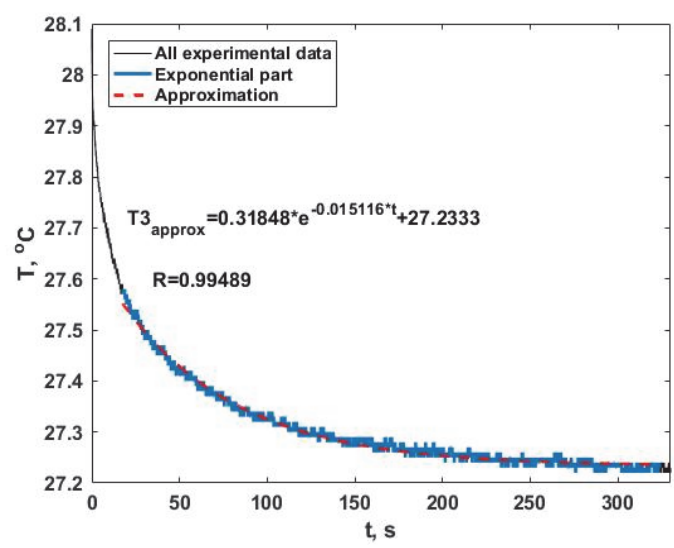

b)

Figure 2: (a) IR image of the specimen during pulse point heating; (b) Approximation of the cooling curve of the specimen after its pulse point heating. 
Fig. 3 shows the infrared imaging of the specimen surface at the final stage of loading before its fracture and the timetemperature dependence during the test. At the beginning of the test, the average surface temperature decreases due to the thermoelastic effect, then the thermoplastic effect prevails, and the temperature of the specimen increases until the moment of necking and complete failure.

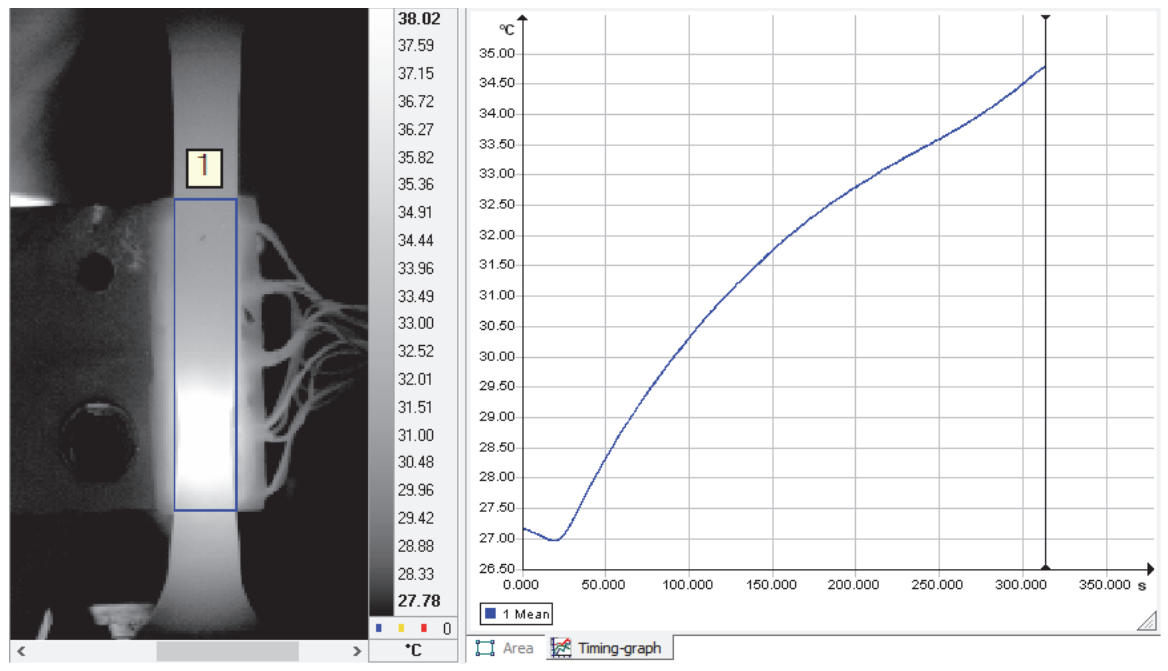

Figure 3: IR image of the specimen under quasistatic tensile conditions.

According to Eqn. (4), the time dependence of the heat source field was estimated based on the change of the specimen temperature during the mechanical test (Fig. 4a). The calculated heat source power data were verified by analyzing the data recorded by the Seebeck effect-based heat flux sensor. The heat source obtained by both methods do not coincide completely, which can be explained by different sensitivities of the devices, and errors of numerical processing of the infrared thermography data. Nevertheless, these results suggest the possibility of using non-contact measurements to estimate the heat source distribution on the specimen surface.

One part of the irreversible plastic work contributes to heat generation, and another is stored as the energy of crystal defects accompanying plastic deformation, known as the stored energy of cold work. For flat specimens, the plastic work spent on deformation of the specimen can be defined as a function of strain rate $V$ and loading force $F(t)$ :

$$
W_{p}(t)=F(t) V
$$

The stored energy is determined as a difference between the plastic work spent on deformation and the integral heat dissipation. The time dependences of these values are presented in Fig. 4b. Analysis of the data in Fig. 4b suggests that, when the material approaches fracture, the value of stored energy in the material reaches a critical value, and the rate of stored energy tends towards zero.

In work [10], the algorithm allowing estimation of the heat transferred by convection, conduction and radiation:

$$
\int_{V}\left(\oint \sigma_{i j} \cdot d \varepsilon_{i j}\right) \cdot d V=\int_{V}-\lambda \cdot \nabla^{2} T \cdot d V+\int_{S_{c \nu}} \alpha \cdot\left(T-T_{\infty}\right) \cdot d S_{c v}+\int_{S_{i r}} \kappa \cdot \sigma_{n} \cdot\left(T^{4}-T_{\infty}^{4}\right) \cdot d S_{i r}+\int_{V}\left(\rho \cdot c \cdot \frac{\partial T}{\partial t}+\dot{E}_{p}\right) \cdot d V
$$

where $\lambda$ is the thermal conductivity of the material, $\alpha$ is the heat transfer coefficient by convection, $\kappa$ is the surface emissivity, $\rho$ is the density, $c$ is the specific heat, $\sigma_{n}$ is the Stephan-Boltzmann constant equal to $5.67 \cdot 10^{8} \mathrm{~W} /\left(\mathrm{m}^{2} \mathrm{~K}^{4}\right)$, $\dot{E}_{p}$ is the rate of variation of the stored energy, $T_{\infty}$ is the room temperature, $T(x, y, z, t)$ is the time-dependent temperature field, and $S_{c v}, S_{c d}, S_{i r}$ are the three parts of the external surface of the control volume $V$ through which the heat $Q$ is transferred by convection, conduction and radiation, respectively. 


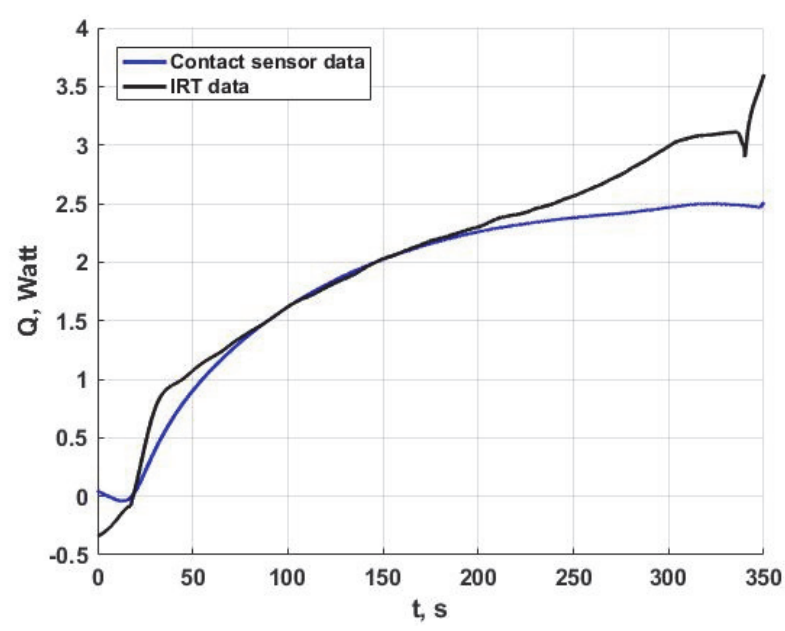

a)

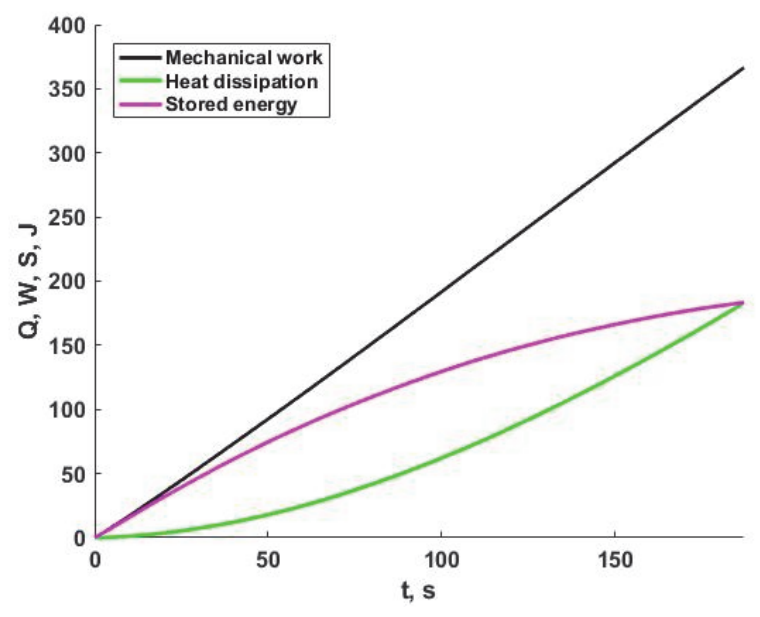

b)

Figure 4: (a) Infrared thermography data vs heat flux sensor data; (b) Time dependence of heat dissipation and stored energy (Eqn. 4).

The parameters $\mathcal{Q}$ and $\dot{E}_{p}$ can be derived via Eqn. (6) using the experimental surface temperature measurements and the room temperature data, provided that the heat transfer coefficient $\alpha$ and the surface emissivities $\kappa$ are known.

In our case, the specimen surface was coated with a thin layer of amorphous carbon, and the emissivity coefficient was equal to 0.92 . The following three different formulae were used in order to evaluate the heat transfer coefficient in the case of natural convection [11]:

$$
\begin{aligned}
& N u=\left\{0.825+\frac{0.387 \cdot R a^{1 / 6}}{\left[1+\left(\frac{0.492}{P r}\right)^{9 / 16}\right]^{8 / 27}}\right\}^{2}, \\
& N u=A \cdot(G r \cdot P r)^{1 / 4}, \quad A^{4}=\frac{P r}{2.43478+4.884 \cdot P r^{1 / 2}+4.95283 \cdot P r}, \\
& N u=0.667 \cdot\left(\frac{P r}{0.952+P r}\right)^{1 / 4} \cdot R a^{1 / 4}, \\
& N u=\frac{\alpha \cdot L}{\lambda_{a}},
\end{aligned}
$$

where $G r, N u, P r, R a$ are the non-dimensional Grashof, Nusselt, Prandtl and Rayleigh numbers, $L$ is the length of the examined part of the specimen, and $\lambda_{a}$ is the thermal conductivity of the air $[\mathrm{W} /(\mathrm{m} \mathrm{K})]$.

It has been established that these formulae give very similar results and the mean value of heat transfer coefficient $\alpha=3.7$ $\mathrm{W} /\left(\mathrm{m}^{2} \mathrm{~K}\right)$.

Based on the obtained results, we can conclude that conduction is the predominant heat transfer mechanism for titanium alloy Grade 2 specimens (Fig. 5a). The curve describing stored energy evolution during the mechanical test is presented in Fig. 5b. This plot coincides with the curve obtained according to Eqn. (4), which confirms the possibility of using the stored energy value as a reliable parameter for diagnosis of fracture.

\section{CONCLUSION}

volution of irreversible deformation in the material is accompanied by the processes of energy accumulation and dissipation. In this work, we analyzed the energy balance of the material during the deformation process by two methods: infrared thermography technique and the method which uses the heat flux sensor. The infrared scanning 
method was found to be efficient for estimating the accumulated energy in the titanium alloy Grade 2. It was established that conduction is the predominant heat transfer mechanism for the examined material.

The value of stored energy reaches a critical value and stored energy rate tends towards zero at the end of deformation process. This indicates that most of the plastic work is converted into heat, and the material is close to being fractured. Hence, it can be concluded that the analysis of the energy stored in the material can provide an adequate description of damage evolution in metals under deformation.

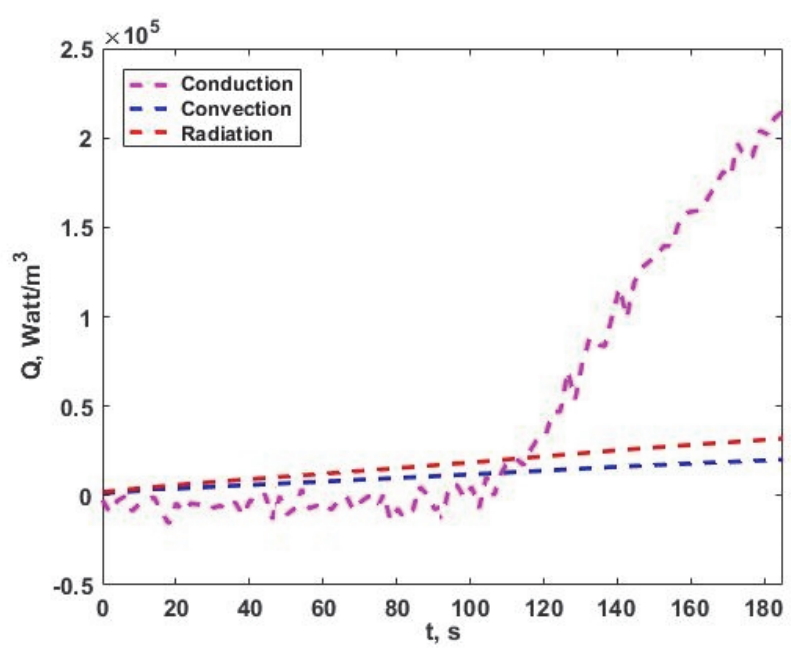

a)

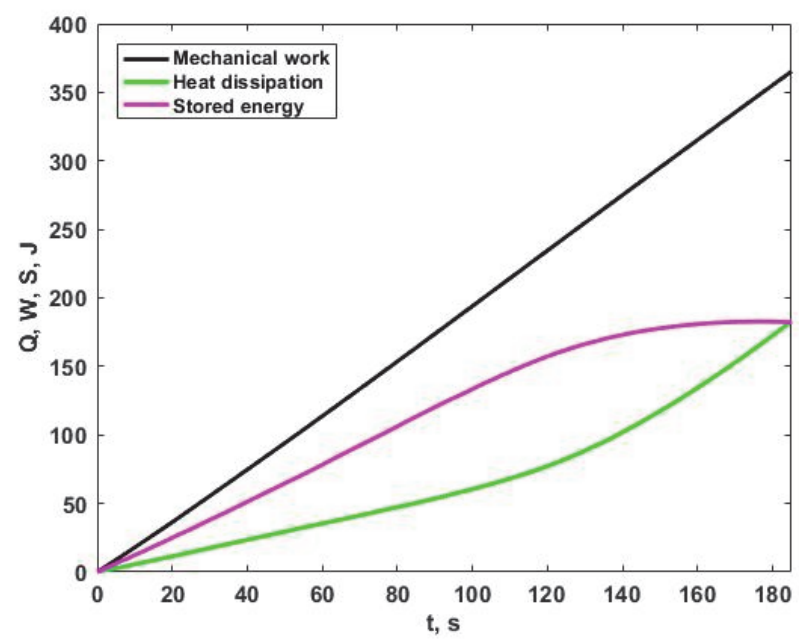

b)

Figure 5: (a) The different contributions of conduction, convection and radiation to the total energy dissipation; (b) Time dependence of heat dissipation and stored energy (Eqn. 5).

\section{ACKNOWLEDGMENTS}

his work was supported by the grants RFBR №18-31-00293 and №16-51-48003.

\section{REFERENCES}

[1] Rosakis, P., Rosakis, A.J., Ravichandran, G., Hodowany J. (2000). A thermodynamic internal variable model for the partition of plastic work into heat and stored energy in metals, J. Mech. Phys. Solids, 48, pp. 581-607.

[2] La Rosa, G., Risitano, A. (2000). Thermographic methodology for rapid determination of the fatigue limit of materials and mechanical components, // Int. J. Fatigue, 22, pp. 65-73. DOI: 10.1016/S0142-1123(99)00088-2.

[3] Oliferuk, W., Maj, M., Raniecki B. (2004). Experimental analysis of energy storage rate components during tensile deformation of polycrystals, Mater. Sci. Eng., 374, pp. 77-81. DOI: 10.1016/j.msea.2003.12.056.

[4] Benaarbiaa, A., Chrysochoos, A., Gilles R. (2014). Kinetics of stored and dissipated energies

[5] Risitano A., Risitano G. (2010). Cumulative damage evaluation of steel using infrared thermography, Theor. Appl. Fract. Mec., 54(2), pp. 82-90. DOI: 10.1016/j.tafmec.2010.10.002.

[6] Plekhov, O.A., Saintier, N., Naimark, O.B. (2007). Experimental study of energy accumulation and dissipation in iron in an elastic-plastic transition, Tech. Phys. Lett., 52(9), pp. 1236-1238. DOI: 10.1134/S106378420709023X.

[7] Fedorova, A., Bannikov, M., Terekhina, A., Plekhov, O. (2014). Heat dissipation energy under fatigue based on infrared data processing, Quant Infrared Thermogr J., 11(1), pp. 2-9. DOI: 10.1080/17686733.2013.852416.

[8] Iziumova, A.Yu., Vshivkov, A.N., Prokhorov, A.E., Plekhov, O.A., Venkatraman, B. (2016). Study of heat source evolution during elastic-plastic deformation of titanium alloy Ti-0.8Al-0.8Mn based on contact and non-contact measurements, PNRPU Mechanics Bulletin, 1, pp. 68-81. DOI: 10.15593/perm.mech/2016.1.05.

[9] Iziumova, A.Yu., Plekhov, O.A., Vshivkov, A.N., Prokhorov, A.A., Uvarov, S.V. (2014). Studying the Rate of Heat 
Dissipation at the Vertex of a Fatigue Crack, Tech. Phys. Lett., 40, pp. 830-832. DOI: 10.1134/S1063785014090211. [10] Meneghetti, G. (2007). Analysis of the fatigue strength of a stainless steel based on the energy dissipation, Int J Fatigue, 29, pp. 81-94. DOI:10.1016/j.ijfatigue.2006.02.043

[11] Bonacina, C, Cavallini, A, Mattarolo, L. (1992). Heat transfer, Cleup Editore, Padova. 